\title{
Kidney microbiome in patients with kidney carcinoma: Role of SA and SNZ gene expression
}

\section{Type}

Research paper

\section{Keywords}

microbiome, renal cell carcinoma, kidney tumor, SA, SNZ

\begin{abstract}
Introduction

A kidney tumor is among the 10 most common cancers. Among kidney tumors, renal cell carcinoma (RCC) is one of the most common types with an alarming increasing incidence rate. Although the disruption of microbiota is an established factor in the progression of intestinal cancers, its role in other types of cancers has been under-studied.
\end{abstract}

Material and methods

In this study, the microbiome disruption and the involvement of SNZ (SCHNARCHZAPFEN) and SA (Stromalin) genes in the development of kidney cancer have been focused on using a combination of genetic and bioinformatic analysis. The microbiomes of kidney tumor patients were analyzed using various genetic and bioinformatic variations. Genetic and bioinformatic analyses were performed to identify operational taxonomic units (OTUs), SNZ, SA, and annotate species were determined using 41 samples from a population of kidney tumors.

\section{Results}

The whole samples from the kidney tumor of patients were screened by PCR amplification and a total of 1317 OTUs were identified. Among them, 379 were common among the two populations, 766 were unique to the SA gene, and 172 to SNZ. SA was more abundant in Gammaproteobacteria and bacilli, while SNZ had a higher abundance in bacteroidia and actinobacteria. Correlation analysis was performed to find out the bacteria that were differentially expressed among the population samples.

\section{Conclusions}

To sum up, our study reveals that SA and SNZ are differentially expressed in the microbiome of the kidney tumor that is associated with the development of kidney tumors such as renal cell carcinoma in human populations. 


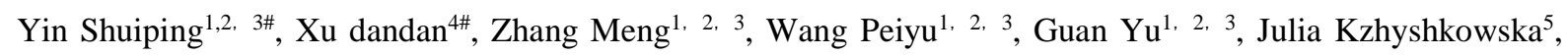

1. Department of Urology, The First Affiliated Hospital of Anhui Medical University; 230022

2. Institute of Urology, Anhui Medical University, Hefei, Anhui, China.

3. Anhui Province Key Laboratory of Genitourinary Diseases, Anhui Medical University, Hefei, Anhui, China.

\#: These authors contributed equally. 


\section{Abstract}

\section{Introduction}

A kidney tumor is among the 10 most common cancers. Among kidney tumors, renal cell carcinoma (RCC) is one of the most common types with an alarming increasing incidence rate. Although the disruption of microbiota is an established factor in the progression of intestinal cancers, its role in other types of cancers has been under-studied.

\section{Materials and methods}

In this study, the microbiome disruption and the involvement of SNZ (SCHNARCHZAPFEN) and SA (Stromalin) genes in the development of kidney cancer have been focused on using a combination of genetic and bioinformatic analysis. The microbiomes of kidney tumor patients were analyzed using various genetic and bioinformatic variations. Genetic and bioinformatic analyses were performed to identify operational taxonomic units (OTUs), SNZ, SA, and annotate species were determined using 41 samples from a population of kidney tumors.

\section{Results}

The whole samples from the kidney tumor of patients were screened by PCR amplification and a total of 1317 OTUs were identified. Among them, 379 were common among the two populations, 766 were unique to the SA gene, and 172 to SNZ. SA was more abundant in Gammaproteobacteria and bacilli, while SNZ had a higher abundance in bacteroidia and actinobacteria. Correlation analysis was performed to find out the bacteria that were differentially expressed among the population samples.

\section{Conclusion}


48 To sum up, our study reveals that SA and SNZ are differentially expressed in the microbiome

49 of the kidney tumor that is associated with the development of kidney tumors such as renal cell

50 carcinoma in human populations.

51 Keywords: kidney tumor, SNZ, SA, microbiome, renal cell carcinoma.

52

53

54

55

56

57

58

59

60

61

62

63

64

65

66

67 
69

Kidney cancer is the $13^{\text {th }}$ most common cancer type with a prevalence of $2.4 \%$, and about 330000 new cases are reported every year [1]. The conditions are graver in industrialized countries, where it is the $7^{\text {th }}$ most predominant cancer type $[1,2]$. It is a more prevalent tumor during the early fifties and is the major mortality cause [3]. Renal cell carcinoma (RCC) is one of the most common types of cancer with an increasing incidence, accounting for over 400,000 new cases diagnosed and approximately 175,000 deaths globally each year [2]. Nearly 30\% of patients are diagnosed at an advanced or metastatic stage with an estimated 5-year survival rate of $10 \%$ [3]. Though the development of targeted agents and immune checkpoint inhibitors has provided dramatic clinical benefits to the patients over the past few decades, a better understanding of the RCC pathogenesis will still be necessary to further reduce the mortality rate [4].

Consistent studies suggested various triggering factors can lead to kidney cancer and chronic kidney disease $[5,6]$. Moreover, any imbalance in the kidney microbiome can also predispose to fatal conditions such as renal cell carcinoma, and such a biochemical milieu can greatly influence the structure, composition, and role of microbial flora [7]. Furthermore, the normal microbiome has beneficial effects; however, the microbiota composition is significantly altered in carcinomic conditions. This disturbance in microflora creates abnormal conditions such as the production of harmful byproducts and inflammatory cytokines, which significantly contribute to chronic kidney diseases [24]. Consistently, the relative role of dysbiosis in other disease conditions such as gastrointestinal cancers has been demonstrated previously [8-10]. Recently, it was found that any disturbances in the intestinal barrier can lead to translocation of bacteria, which then persistently triggers systematic inflammation and exacerbate chronic kidney diseases $[11,12]$. Furthermore, certain therapeutic interventions or conditions like hyperkalemia can greatly modify the luminal microbial milieu and such modification may 
substantiate the gut epithelial barrier. Consequently, the microbial antigens can leak into the systemic circulation [13, 14]. Likely, vitamin D deficiency is frequently associated with microbiome dysbiosis, with consequent increases in bactericides and proteobacteria phyla [15]. Research into the gut microbiome and vitamin $\mathrm{D}$ is therefore considered promising for understanding, treating, and preventing autoimmune and allergic diseases [16]. Furthermore, recent studies proposed that chronic inflammations could contribute to the progression and development of prostate cancer $[17,18]$. Moreover, the role of vascular endothelial growth factor (VEGF) production in allergy and systemic lupus erythematosus (SLE) confirmed the key role of VEGF in angiogenesis in cancer patients ad in patients with inflammatory chronic immune-mediated diseases [19]. Several studies propose that the microbial inhabitants of the urinary tract are distinct from those harboring other parts of the body even in disease conditions [20]. For instance, kidney cystitis shares a common microbiome with kidney cancer [21]. Moreover, the relative role of microbiome kidney cancer needs further to be elucidated. Lately, intestinal microbiome research has arisen in the field of kidney disease, for which various research approaches are employed, such as conventional incubation, shotgun metagenomic sequencing, bacterial 16S ribosomal RNA (rRNA) Illumina MiSeq sequencing, and many others [22].

The relative role of the gene in various cancer pathogenesis has been previously investigated [23]. In the current study, we have investigated how the kidney microbiome can differentially express SNZ (SCHNARCHZAPFEN) and SA (Stromalin), which may contribute to the progression of kidney tumors. SNZ gene is from AP2/ERF transcription factor AP2 (Floral Homeotic Protein Apetala 2) family encoding AP2-like ethylene-responsive transcription factor SNZ protein. It acts as a transcription activator and binds GCC-box pathogenesis-related promoter elements on DNA. It has been genetically linked to various pathological conditions such as blood pressure. Recent reports demonstrate that high throughput sequencing-HTS (hu 
118 li tai shao) has proved to be a powerful tool in analyzing dominant and subdominant 119 populations of microbes and their dynamics in various pathophysiological complexities [24, 25]. The product of the SA gene, despite lacking significant homology with other proteins amino acid sequences, has been reported in various studies. The main function of the gene 122 product associated with the SA gene remains unclear. Ishinaga et al. showed that SA gene expression in the kidney is associated with the pathogenesis of hypertension [26].

In the present study, we utilized $16 \mathrm{~S}$ rRNA gene sequencing to characterize the kidney microbiome potentially associated with kidney cancer. Our research aimed to analyze 41 samples from patients with kidney carcinoma for differential expression of SA and SNZ in kidney microbiome populations and to rule out dysbiosis having a higher abundance in kidney cancer patients. Further, we aimed to determine whether the microbiome is significantly different between these groups and to predict functional pathways that are significantly enriched in the kidney microbiome.

\section{Methods}

\section{Recruitment of patient population.}

All the specimens were obtained from the patients under the standard and approved protocol. The patients who showed a willingness to participate in the study were added to the current study. Kidney urine and biopsy were collected from patients who were diagnosed with kidney carcinoma. To avoid confounding factors that may affect the kidney microbiome, strict parameters were selected for exclusion criteria. Patients with kidney stones or on antibiotic therapy for the last one month, patients with hydronephrosis, congenital comorbidities, history of major kidney surgery, autoimmune diseases, or patients with diabetes were excluded from the current study. 


\section{Samples collection and DNA isolation and processing}

144 Samples of kidney carcinoma and cysts were collected using catheterization via transurethral route using the aseptic technique. The samples were collected before any use of antibiotics, which may negatively affect the microbiome at $-80{ }^{\circ} \mathrm{C}$. The samples were pelleted and then centrifuged at $1200 \mathrm{~g}$ for at least $10 \mathrm{~min}$ at $4{ }^{\circ} \mathrm{C}$. The pellets were resuspended in DNA free total volume of $500 \mathrm{ul}$ of $1 \mathrm{xPBS}$, and then genomic DNA was extracted for all samples through the DNeasy Water Kit. DNA was quantified using Qubit (Life Technologies, USA). The extracted sample was then stored at $-20{ }^{\circ} \mathrm{C}$ until further experimentations.

\section{S rRNA gene library generation and HiSeq sequencing}

The V5 hypervariable region of the 16S rRNA gene was amplified through a polymerase chain reaction. The following conditions were used to run the process: First denaturation stage of 30 cycles at $98 \mathrm{C}$ for 45 seconds. It was followed by the annealing stage at $55{ }^{\circ} \mathrm{C}$ for 45 seconds and then the elongation stage at $72{ }^{\circ} \mathrm{C}$ for 45 seconds. The final step was the extension at 72 bullet blended beads was used as a negative control during processing.

\section{Universal 16S rDNA real-time PCR.}

162 A universal 16S rDNA quantitative real-time PCR (qPCR) assay was developed using the first round V6 primer set. The number of $16 \mathrm{~S}$ rDNA copies was quantified to a standard curve of known copies of Escherichia coli DNA.

\section{Bioinformatic analysis}


Sequencing data was processed to exclude any low-quality reads and polluted adapter using

Mothur. The clean reads were then merged to tags using FLASH software, then assigning OUTs based on USEARCH $97 \%$ sequence similarity. Using the RDP classifier, the representative sequences of each OUT were taxonomically classified based on the green gene database. The threshold for sequence positivity was set at a cutoff of 2000 sequence reads.

The analysis was performed to find the differentially representative among the sample groups by LEfSe software. The threshold on the logarithmic LDA score for discriminative features was 2. Bacterial community functional pathways were inferred and imported by using the PICRUSt algorithm while functional predictions were performed using the Kyoto Encyclopedia of Genes and Genomes (KEGG) orthology.

\section{Results}

\section{Sequencing data and biodiversity of kidney microbiome}

A total of 41 kidney carcinomic samples were sequenced into 50000 reads. The reads were classified into 1317 unique operational taxonomic units (OTUs) with a 97\% similarity level (Fig.1A, 1B). Our results showed that the SA gene has the highest OTUs comprising a total number of 766 while substantial overlap in OTUs was observed between SA and SNZ (Fig. 1C). The rarefaction curve analysis demonstrated the sufficiency of our read coverage to capture sample diversity (Figure. S1). SA is present in more abundance in gammaproteobacteria and bacilli, while SNZ was found in actinobacteria and bacteroidia. For $\alpha$ - diversity, Good coverage index values of all libraries were above (99\%). The $\alpha$ - diversity indices, including observed species, Chao 1 index, ACE index, Shannon diversity index, of the microbiota having the SA gene, were all higher than those of the SNZ containing group (Fig. 1D). Moreover, significant differences were observed in the Chao and ACE indices between 
SA and SNZ containing microbiota. The other $\alpha$ - diversity of kidney microbiota was also evaluated, and all indices showed no significant difference for Good's coverage index observed species, Shannon diversity index. For $\beta$ - diversity, we applied unweighted and weighted principal coordinate analysis (PCoA) to display discrepancies among the two genes. It showed that SA and SNZ samples clustered closer in proximity to each other (Fig. 1E). We further performed an analysis of similarities (ANOSIM) and found that the kidney microbiota structure was significantly different for SA and SNZ genes (ANOSIM, P < 0.05).

\section{Effect of cancer on OTUs abundance profiles in the different habitats}

A PLS-DA was performed to identify the OTUs that contributed the most to the variance between samples. The bacterial composition for SA and SNZ are closely related (Fig. 2A). A heat map shows that a majority of these OTUs decreased in relative abundance in the SA compared with the SNZ (Fig. 2B). Network analysis between SA and SNZ samples were colored in light blue, light green, and light red. The circular dots correspond to OTUs. The node size is proportional to the number of edges (lines connecting the nodes). The OTUs detected in at least two samples of the same habitat were conserve (Fig. 2C).

\section{Taxonomic analysis of kidney microbiota composition}

To identify the differentially represented taxa in kidney carcinoma cells, we compared the relative abundance of microbiota for SA and SNZ distribution at different taxonomic levels. Phylotypes with a median relative abundance larger than $0.001 \%$ of the total abundance in either for SA or SNZ gene were included for comparison. At the phylum level (Fig. 3A), a significant difference was observed between these two groups in the average abundance of Firmicutes (bacilli), Bacteroidetes, Proteobacteria, and actinobacteria. Gammaproteobacteria 
and bacilli were found showing SA affinity, while actinobacteria and bacteroidia had a higher SNZ (Fig. S2). Of interest, at the phylum or class level, the overall bacterial compositions for SA and SNZ were quite similar (Fig. 3A, 3B). However, a few taxa are differentially represented in these two groups at other taxonomic levels (Fig. 3C).

\section{Specific urinary genera associated with kidney carcinoma}

To confirm the differentially abundant taxa for SA and SNZ in kidney carcinoma, we further applied LEfSe, a software using an algorithm for high-dimensional biomarker discovery. The microbiome biomarker discovery approach called linear discriminate analysis coupled with effect size measurements (LEfSe) revealed that certain strains were enriched either for SA or SNZ. Only taxa with logarithmic, linear discriminant analysis (LDA) score more than 2.0 and $\mathrm{P}<0.05$ in the Wilcoxon test were considered differentially represented. LEfSe identified certain discriminative features with significantly different relative abundance among SA and SNZ (Fig.4A, 4B).

\section{Potential functional pathways associated with kidney carcinoma}

Having observed a distinct kidney microbiota in kidney carcinoma, we further evaluated whether the different bacterial community was associated with specific alterations involved in metabolic processes. The functional pathways of the kidney microbiome for SA and SNZ were inferred using the PICRUSt tool (Fig.5A). Meanwhile, the predicted metabolic pathway showed an equal distribution for SA and SNZ gene containing microbiota, and the relative distribution is shown (Fig.5B, 5C, 5D).

\section{Discussion}

In the present study, 16S rRNA gene sequencing and bioinformatics tools were used to compare the microbiome of kidney cancer cells for the relative distribution of two different genes, i.e., SA and SNZ. We tried to seek out the differences in the residing microbiome, which may affect 
relatively kidney cancer. Our results showed significantly reduced species diversity and altered microbial profiles. Several differentially represented taxa and functional pathways were found that showed differential expression for SA and SNZ. Besides, we found that the overall bacterial composition and predicted functional pathways of both populations were similar. Kidney carcinoma is the most prevalent tumor type with different pathologies and contributing factors [27].

Several consistent studies validated the relative role and occurrence of kidney microbiomes in various associated conditions [28, 29]. Moreover, the mechanisms that certain bacteria behave differently have been previously well documented [30]. However, the potential mechanism that bacteria contribute to kidney carcinoma remains obscure. The role of the healthy microbiome on kidney cancer has not been studied to date. However, several studies suggested an association between viral infections and the risk of kidney cancer [31], yet these remain controversial and at times contradictory to each other [31]. Several mechanisms are proposed, including that bacteria may alter the flora of kidney tissue-prone kidney tissue to foreign invaders [32].

16S rRNA gene sequencing followed by bioinformatics analysis was done to determine the specificity of SA and SNZ for kidney microbiomes in kidney carcinoma. As the microbiome is not only related to the symbiotic relationship but it can also adversely contribute to certain diseases and cancer [20]. In the human microbiome, firmicutes and proteobacteria are highly dominant, along with bacteroidetes and actinobacteria [33]. In our study, we found that there is minor diversity in species and microbial profile in the kidney carcinomic sample. Moreover, several different taxa and functional pathways showing equal distribution for SA and SNZ genes. We demonstrated distinct kidney microbiota in tumor cells. Our results showed that kidney carcinoma patients had significantly lower species diversity. According to previous literature, decreased microbiota diversity was related to inflammation and implicated in 
diseases such as obesity and type II diabetes in some cases [34]. Moreover, we found that several bacterial taxa associated with inflammation were overrepresented or underrepresented in the kidney tissue associated with carcinoma. The most differentially represented taxa at the genus level were Acinetobacter, showing SA and SNZ gene distribution. As opportunistic pathogens, proteobacteria, actinobacteria, and bacteroidia are associated with kidney infection in individuals with underlying medical risk factors, such as diabetes mellitus and immunosuppression [35-39]. Interestingly, the abundance of proteobacteria, actinobacteria, and bacteroidia were higher in the SNZ and SA. These bacteria are considered commensal bacteria and colonize the gastrointestinal tract, vaginal tract, and urinary tract. It could synthesize short-chain fatty acids, which could protect against inflammation in acute kidney injury [40]. The decreased level of Prevotella favors inflammatory processes and has been implicated in several pathological conditions, including type 2 diabetes, diabetic nephropathy, and chronic prostatitis [41].

Another important finding in this study was the similarity of overall bacterial composition for SA and SNZ distribution. Traditionally, bacteria are considered to access the upper urinary tract under certain conditions, such as urinary reflux or bacteria translocation in severe systemic diseases. However, a preliminary study showed that bacteria could be detected in the upper urinary tract of kidney stone patients without urinary tract infections [42]. Besides, our PICRUSt results showed no significant difference in the predicted functional pathways between SA and SNZ. Meanwhile, we also noted that a few taxa were differentially represented in another group, remaining an area for future research.

In summary, our results revealed significantly decreased species diversity, enrichments of proinflammatory bacteria, and underrepresentation of anti-inflammatory taxa in the kidney microbiota of carcinoma. We also predicted several functional pathways that were significantly enriched in the kidney microbiome of infected patients. Among these pathways, bacterial 
metabolism is a key regulator of the cell membrane and has been demonstrated as an entrance gate in bacteria-host interactions [43].

291 Some limitations should be noted when interpreting our results. First, all participants were

292 Chinese and the sample size is relatively small, limiting generalizability and comparison of SA and SNZ.

\section{Future Perspective}

Further large-scale studies are needed to investigate the kidney microbiota across ethnicity

types and other types of involved genes to verify the role of SA and SNZ in kidney tumor.

\section{Conflict of interest:}

None

299

Funding

300

None

301

\section{Availability of data}

302

All the data can be requested from the corresponding author upon reasonable request

\section{Ethical and security considerations}

Not applicable due to the nature of bioinformatics study.

\section{References}

[1] G. Scelo and T. L. Larose, "Epidemiology and risk factors for kidney cancer," Journal of Clinical Oncology, vol. 36, p. 3574, 2018.

[2] R. R. Kotecha, R. J. Motzer, and M. H. Voss, "Towards individualized therapy for metastatic renal cell carcinoma," Nature Reviews Clinical Oncology, vol. 16, pp. 621-633, 2019.

[3] R. L. Siegel, K. D. Miller, and A. Jemal, "Cancer statistics, 2019," CA: a cancer journal for clinicians, vol. 69, pp. 7-34, 2019. 
[4] J. J. Huang and J. J. Hsieh, "The therapeutic landscape of renal cell carcinoma: from the dark age to the golden age," in Seminars in nephrology, 2020, pp. 28-41.

[5] J. Malyszko, P. Tesarova, G. Capasso, and A. Capasso, "The link between kidney disease and cancer: complications and treatment," The Lancet, vol. 396, pp. 277-287, 2020.

[6] B. Stengel, "Chronic kidney disease and cancer: a troubling connection," Journal of nephrology, vol. 23, p. 253, 2010.

[7] S. Al Khodor and I. F. Shatat, "Gut microbiome and kidney disease: a bidirectional relationship," Pediatric Nephrology, vol. 32, pp. 921-931, 2017.

[8] S. Zou, L. Fang, and M.-H. Lee, "Dysbiosis of gut microbiota in promoting the development of colorectal cancer," Gastroenterology report, vol. 6, pp. 1-12, 2018.

[9] A. P. Bhatt, M. R. Redinbo, and S. J. Bultman, "The role of the microbiome in cancer development and therapy," CA: a cancer journal for clinicians, vol. 67, pp. 326-344, 2017.

[10] F. A. Shaieb, A. A. Issa, and A. Meragaa, "Antimicrobial activity of crude extracts of cyanobacteria Nostoc commune and Spirulina platensis," Archives of Biomedical Sciences, vol. 2, pp. 34-41, 2014.

[11] H. Tilg, N. Zmora, T. E. Adolph, and E. Elinav, "The intestinal microbiota fuelling metabolic inflammation," Nature Reviews Immunology, vol. 20, pp. 40-54, 2020.

[12] A. Sabatino, G. Regolisti, I. Brusasco, A. Cabassi, S. Morabito, and E. Fiaccadori, "Alterations of intestinal barrier and microbiota in chronic kidney disease," Nephrology Dialysis Transplantation, vol. 30, pp. 924-933, 2015.

[13] K. Andersen, M. S. Kesper, J. A. Marschner, L. Konrad, M. Ryu, S. K. VR, et al., "Intestinal dysbiosis, barrier dysfunction, and bacterial translocation account for CKD-related systemic inflammation," Journal of the American society of nephrology, vol. 28, pp. 76-83, 2017.

[14] N. D. Vaziri, J. Wong, M. Pahl, Y. M. Piceno, J. Yuan, T. Z. DeSantis, et al., "Chronic kidney disease alters intestinal microbial flora," Kidney international, vol. 83, pp. 308-315, 2013.

[15] G. Murdaca, A. Gerosa, F. Paladin, L. Petrocchi, S. Banchero, and S. Gangemi, "Vitamin D and Microbiota: Is There a Link with Allergies?," International Journal of Molecular Sciences, vol. 22, p. 4288, 2021.

[16] G. Murdaca, F. Paladin, and S. Gangemi, "Role of Vitamin D in the Clinical Course of Nasal Polyposis," Biomedicines, vol. 9, p. 855, 2021.

[17] K. S. Sfanos, J. Sauvageot, H. L. Fedor, J. D. Dick, A. M. De Marzo, and W. B. Isaacs, "A molecular analysis of prokaryotic and viral DNA sequences in prostate tissue from patients with prostate cancer indicates the presence of multiple and diverse microorganisms," The Prostate, vol. 68, pp. 306-320, 2008.

[18] S. Weiss, Z. Z. Xu, S. Peddada, A. Amir, K. Bittinger, A. Gonzalez, et al., "Normalization and microbial differential abundance strategies depend upon data characteristics," Microbiome, vol. 5, p. 27, 2017.

[19] G. Ciprandi, G. Murdaca, B. M. Colombo, M. De Amici, and G. L. Marseglia, "Serum vascular endothelial growth factor in allergic rhinitis and systemic lupus erythematosus," Human immunology, vol. 69, pp. 510-512, 2008.

[20] M. G. Abernethy, A. Rosenfeld, J. R. White, M. G. Mueller, C. Lewicky-Gaupp, and K. Kenton, "Urinary microbiome and cytokine levels in women with interstitial cystitis," Obstetrics \& Gynecology, vol. 129, pp. 500-506, 2017.

[21] S. H. Berry, M. N. Elliott, M. Suttorp, L. M. Bogart, M. A. Stoto, P. Eggers, et al., "Prevalence of symptoms of bladder pain syndrome/interstitial cystitis among adult females in the United States," The Journal of urology, vol. 186, pp. 540-544, 2011.

[22] H. Mallick, S. Ma, E. A. Franzosa, T. Vatanen, X. C. Morgan, and C. Huttenhower, "Experimental design and quantitative analysis of microbial community multiomics," Genome biology, vol. 18, p. 228, 2017. 
[23] M. H. Bailey, C. Tokheim, E. Porta-Pardo, S. Sengupta, D. Bertrand, A. Weerasinghe, et al., "Comprehensive characterization of cancer driver genes and mutations," Cell, vol. 173, pp. 371-385. e18, 2018.

[24] J. Yin, S. X. Liao, Y. He, S. Wang, G. H. Xia, F. T. Liu, et al., "Dysbiosis of gut microbiota with reduced trimethylamine-N-oxide level in patients with large-artery atherosclerotic stroke or transient ischemic attack," Journal of the American Heart Association, vol. 4, p. e002699, 2015.

[25] V. Senthong, Z. Wang, X. S. Li, Y. Fan, Y. Wu, W. Wilson Tang, et al., "Intestinal microbiotagenerated metabolite trimethylamine-N-oxide and 5-year mortality risk in stable coronary artery disease: the contributory role of intestinal microbiota in a COURAGE-like patient cohort," Journal of the American heart association, vol. 5, p. e002816, 2016.

[26] Y. Ishinaga, T. Nabika, T. Shimada, J. Hiraoka, Y. Nara, and Y. Yamori, "Re-evaluation of the SA gene in spontaneously hypertensive and Wistar-Kyoto rats," Clinical and experimental pharmacology and physiology, vol. 24, pp. 18-22, 1997.

[27] M. C. Markowski, S. A. Boorjian, J. P. Burton, N. M. Hahn, M. A. Ingersoll, S. M. Vareki, et al., "The microbiome and genitourinary cancer: a collaborative review," European Urology, vol. 75, pp. 637-646, 2019.

\section{5, pp. 637-646, 2019.}

[28] Y. Wang, G. Yang, L. You, J. Yang, M. Feng, J. Qiu, et al., "Role of the microbiome in occurrence, development and treatment of pancreatic cancer," Molecular Cancer, vol. 18, pp. 1-13, 2019.

[29] M. Pevsner-Fischer, T. Tuganbaev, M. Meijer, S.-H. Zhang, Z.-R. Zeng, M.-H. Chen, et al., "Role of the microbiome in non-gastrointestinal cancers," World journal of clinical oncology, vol. 7, p. 200, 2016.

[30] C. L. Wells, M. A. Maddaus, and R. L. Simmons, "Proposed mechanisms for the translocation of intestinal bacteria," Reviews of infectious diseases, vol. 10, pp. 958-979, 1988.

[31] H. Abol-Enein, "Infection: is it a cause of bladder cancer?," Scandinavian Journal of Urology and Nephrology, vol. 42, pp. 79-84, 2008.

[32] T. J. Wiles, R. R. Kulesus, and M. A. Mulvey, "Origins and virulence mechanisms of uropathogenic Escherichia coli," Experimental and molecular pathology, vol. 85, pp. 11-19, 2008.

[33] H. E. Jakobsson, C. Jernberg, A. F. Andersson, M. Sjölund-Karlsson, J. K. Jansson, and L. Engstrand, "Short-term antibiotic treatment has differing long-term impacts on the human throat and gut microbiome," Plos one, vol. 5, p. e9836, 2010.

[34] I. Moreno-Indias, F. Cardona, F. J. Tinahones, and M. I. Queipo-Ortuño, "Impact of the gut microbiota on the development of obesity and type 2 diabetes mellitus," Frontiers in microbiology, vol. 5, p. 190, 2014.

[35] A. Hakansson and G. Molin, "Gut microbiota and inflammation," Nutrients, vol. 3, pp. 637-682, 2011.

[36] M. A. Kucher, O. V. Goloschapov, I. S. Moiseev, and B. V. Afanasyev, "Fecal microbiota transplantation as a method to treat complications after hematopoietic stem cell transplantation," Cell Ther Transplant, vol. 6, pp. 20-29, 2017.

[37] M. Villanueva-Millán, "Effects of different antiretroviral treatments on gut microbiota of hivinfected patients," Universidad de La Rioja, 2018.

[38] S. Serrano-Villar, S. Sanchez-Carrillo, A. Talavera-Rodríguez, B. Lelouvier, C. Gutiérrez, A. Vallejo, et al., "Blood Bacterial Profiles Associated with HIV Infection and Immune Recovery," The Journal of Infectious Diseases, 2020.

[39] M. Remely, S. Dworzak, B. Hippe, J. Zwielehner, E. Aumüller, H. Brath, et al., "Abundance and diversity of microbiota in type 2 diabetes and obesity," J Diabetes Metab, vol. 4, p. 2, 2013.

[40] P. B. Mortensen and M. R. Clausen, "Short-chain fatty acids in the human colon: relation to gastrointestinal health and disease," Scandinavian Journal of Gastroenterology, vol. 31, pp. 132-148, 1996. 
A

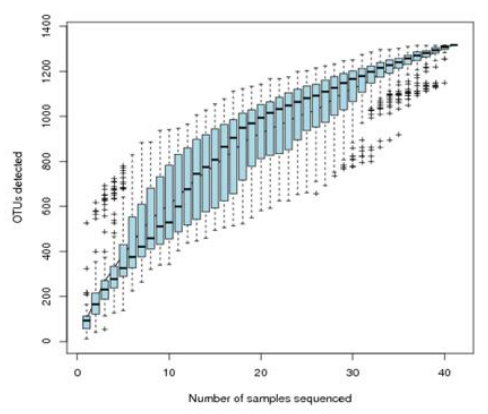

D
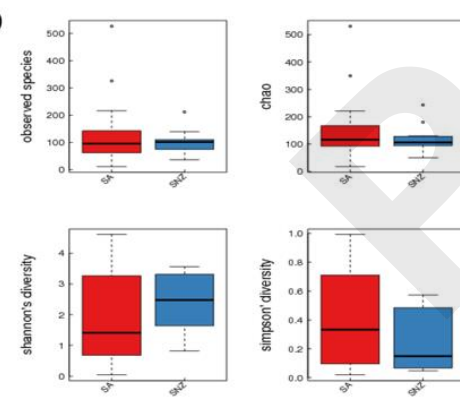

B
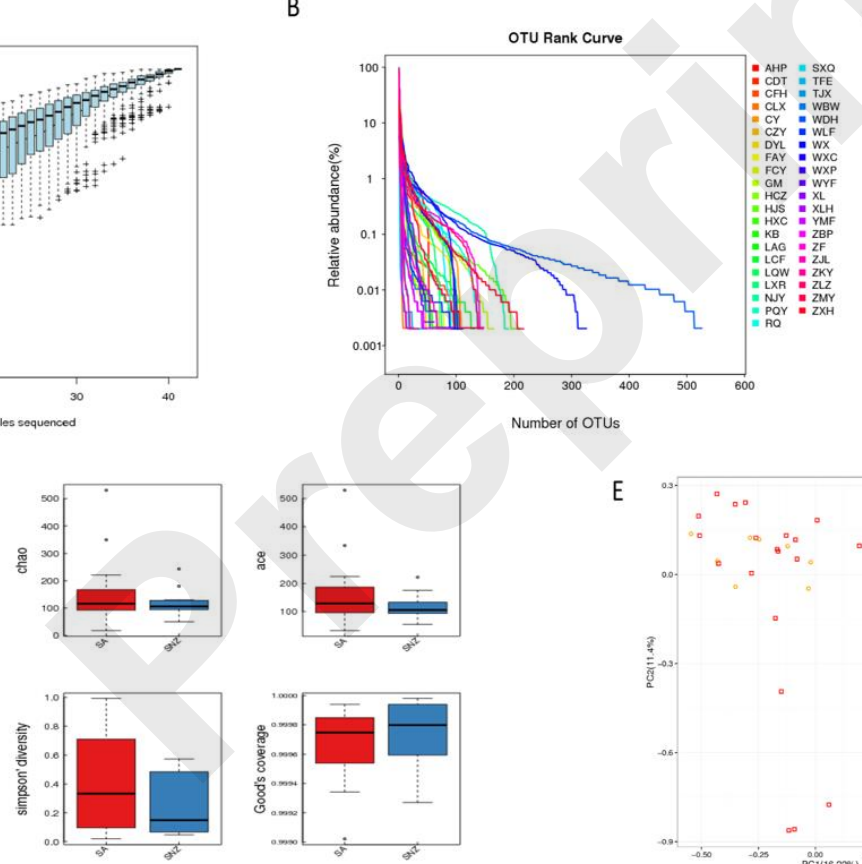
microbiome in men with calcium-based kidney stones," BMC microbiology, vol. 20, pp. 1-10, 2020. relation between urinary tract infections and stone composition in renal stone formers," Scandinavian journal of urology and nephrology, vol. 23, pp. 131-136, 1989.

colonisation and colonisation resistance of the digestive tract part 1: bacteria/host interactions," Microbial ecology in health and disease, vol. 12, pp. 223-239, 2000.

Figure 1: (A) Total number of samples sequenced $(A B)$ and relative abundance. (C) Venn diagram of overlapping OTUs. A total of 1317 OTUs were detected with 766 OTUs in SA, while 172 OTUs were in SNZ samples only, and 379 OTUs in all samples. (D) Microbial $\alpha$ - diversity of urine samples. The $\alpha$-diversity indices include observed species index, Chao 1 index, Ace index, Shannon index, Simpson index, and Good's coverage index. Shannon diversity index and Simpson's diversity index were significantly different between SA and SNZ groups. (E) Microbial diversity analysis. PCoA plot of unweighted and weighted UniFrac metrics for SA (red cube), SNZ (orange circle) groups. 
A

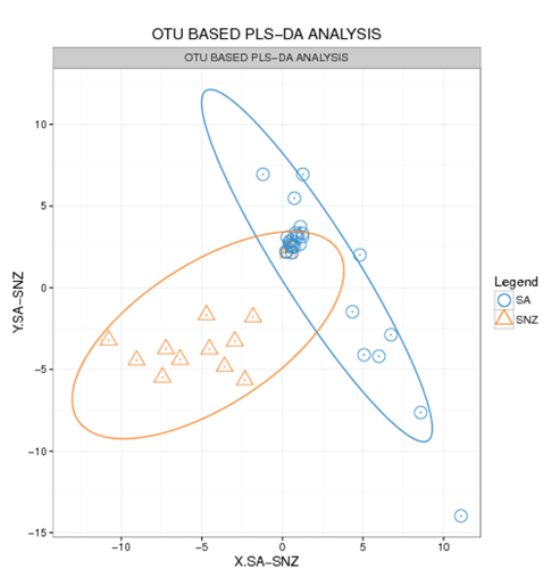

B C

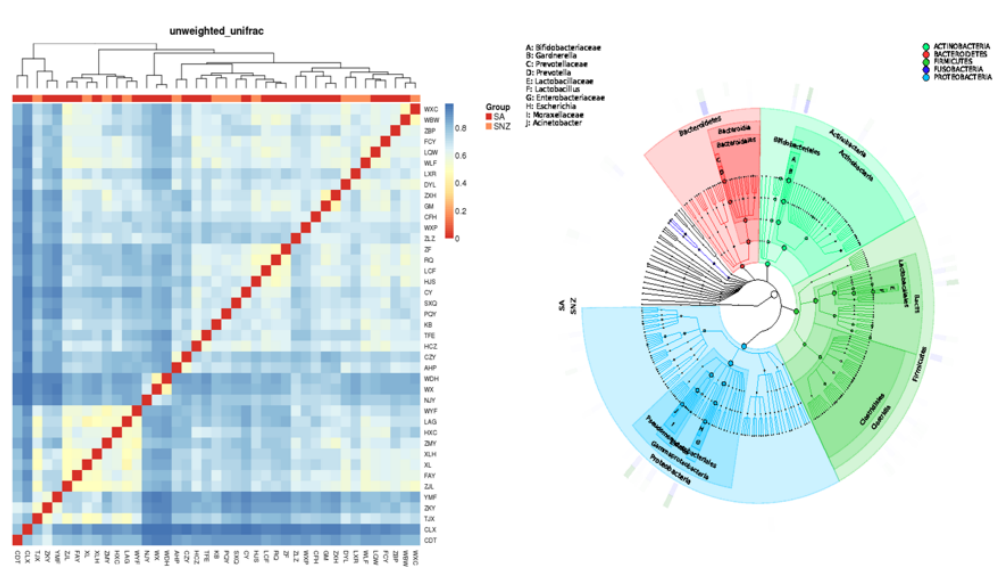

438

439

440

441

442

443

Figure 2: (A) Partial least squares discriminant analysis (PLS-DA) performed on the relative abundance of 1317 OTUs found in 41 samples. (B) Heat map (C) Network analysis.

A

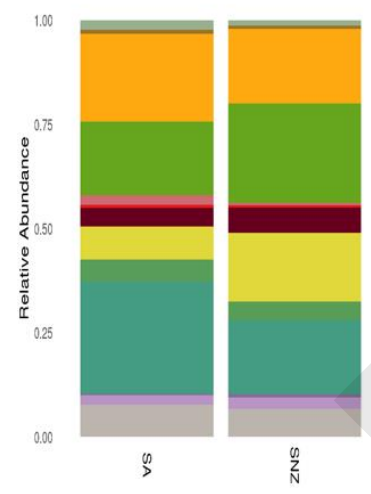

B

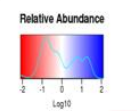

C

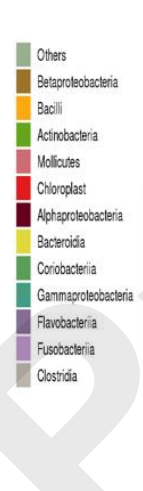

Genus species phylogeny tree

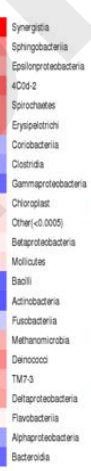

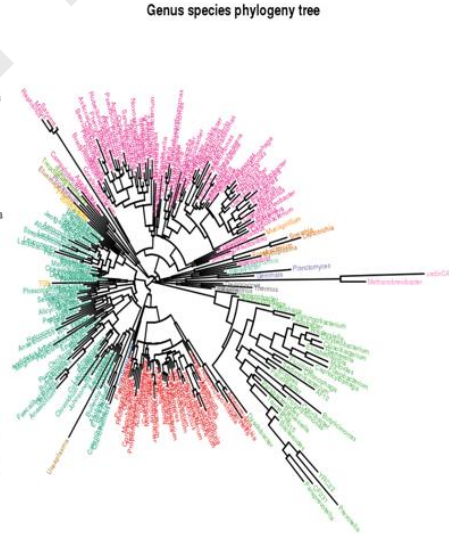

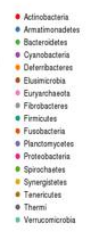

444

445

Figure 3. Bacterial average relative abundance in SA and SNZ groups at different taxonomic levels. (A). Phylum, (B). Order, (C). Genus, Unclassified genera or genera with a relative abundance $<1 \%$ are grouped as "Other".

449 
A

Cladogram

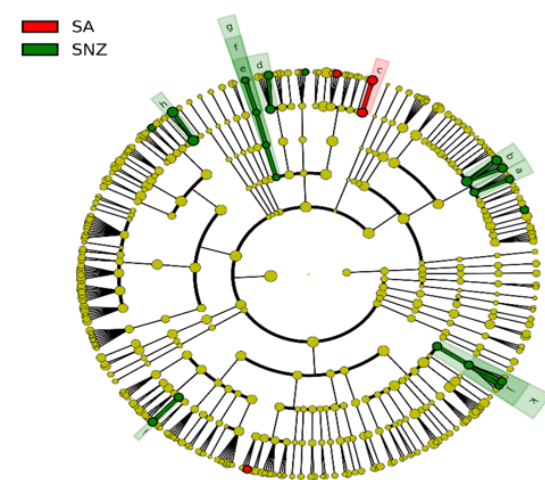

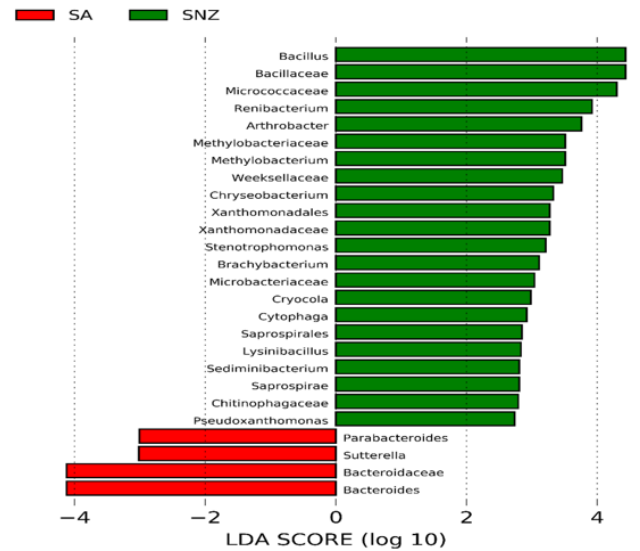

451

452

453

454

455

456

457
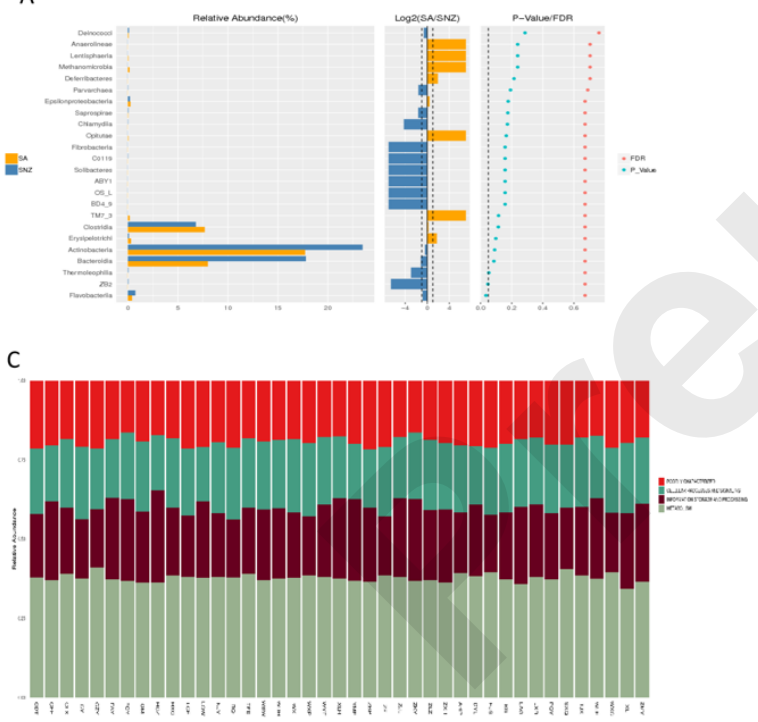
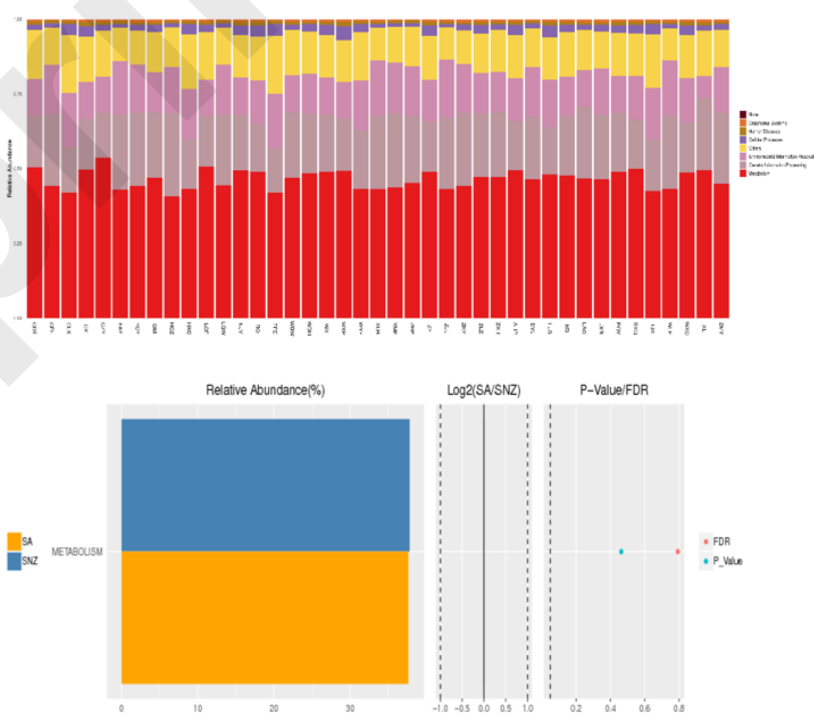

Figure 5. (A) The functional pathways of the kidney microbiome for SA and SNZ were inferred using the PICRUSt tool. (B, C, D) Meanwhile, the predicted metabolic pathway showed an equal distribution for SA and SNZ gene containing microbiota, and the relative distribution is shown. 
The Rarefaction of Samples

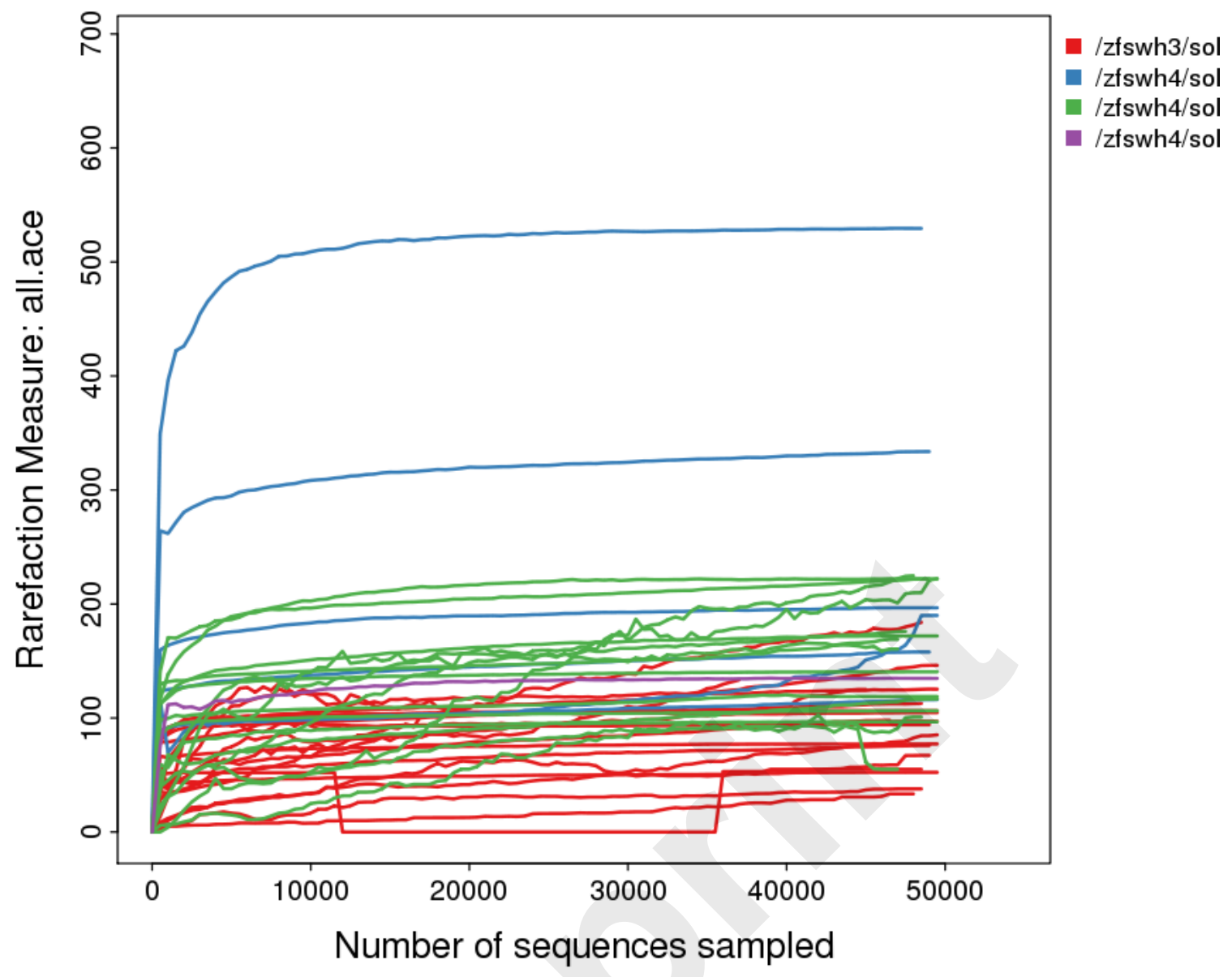

466 Figure S1: The rarefaction curves 